\title{
Second-order torque on the tidal redistribution and the Earth's rotation
}

\author{
S. B. Lambert ${ }^{1, \star}$ and P. M. Mathews ${ }^{2}$ \\ 1 Royal Observatory of Belgium, 1180 Brussels, Belgium \\ e-mail: s.lambert@oma.be \\ 2 Department of Theoretical Physics, University of Madras, Chennai 600025, India
}

Received 14 November 2005 / Accepted 14 March 2006

\section{ABSTRACT}

\begin{abstract}
This study presents a complete treatment of the second order torques on the Earth due to the action of each of the three parts of the degree 2 potential ( $V_{20}$ : zonal; $V_{21}$ : tesseral; and $V_{22}$ : sectorial) on the deformations produced by the other parts, and the consequent effects on nutation. The work of Mathews et al. (2002, J. Geophys. Res., 107, B4) contained a treatment of the action of the tesseral potential on tidal deformations, taking into account the presence of the fluid core, and also of the contributions from mantle anelasticity and ocean tides to the deformations. We extend that work to include the actions of the zonal and sectorial potentials too. Our computations show that an almost complete cancellation takes place between reciprocal contributions; the largest net effect reaches $-35 \mu$ as on the in-phase 18.6-yr nutation in longitude. The total effect found on the precession is 0.1 mas/cy in longitude and in obliquity. The cancellations would have been complete but for the fact that (i) the values of the compliances (deformability parameters) are not the same for deformations excited by the three parts of the degree 2 potential even for a nondissipative Earth and (ii) anelasticity and ocean tides make the contributions to the compliances complex (besides being unequal for the three parts) and thus give rise to out-of-phase components in the response to tidal forcing.
\end{abstract}

Key words. reference systems - Earth

\section{Introduction}

The International Astronomical Union (IAU) has recommended the use of the IAU 2000A nutation model, based on the work of Mathews et al. (2002) (referred to as MHB in the following), from 1 January 2003 in replacement of the IAU 1980 model (see e.g., Seidelmann 1982). The new model takes advantage of the advances of the past decade in the accuracy of theoretical computations on the nutations of the rigid Earth, as well as in the precision of the observational estimates of nutation and precession because of advances in very long baseline interferometry (VLBI) and the increasing length of the VLBI data-set. Truncation levels of the rigid Earth nutation series of Roosbeek \& Dehant (1997), Bretagnon et al. (1997) and Souchay et al. (1999) are all at 0.1 microarcsec ( $\mu$ as) or less. Uncertainties in the VLBI estimates of the prograde and retrograde amplitudes are now in the range from under $10 \mu$ as for nutations of short periods to $100 \mu$ as or less for prominent nutations of long periods. Nevertheless, comparisons of the VLBI nutation time series with IAU $2000 \mathrm{~A}$ reveals rms differences of somewhat under $200 \mu$ as. These residuals are the consequence of various mismodeled or unmodeled influences in the observational strategy as well as in geophysical processes (see e.g., Dehant et al. 2003).

A class of effects only partly taken into account in MHB is due to the action of the degree 2 external and centrifugal potentials on the deformations induced by these potentials. They will be referred to as second-order effects in the following for obvious reasons, since computations of the nutations consider ordinarily the first-order effects, namely, the torque exerted by the

^ On leave from US Naval Observatory, Washington, DC 20392, USA. tesseral tidal potential $V_{21}$ on the ellipsoidal Earth, plus the inertial effects of the deformations produced by $V_{21}$ and by the centrifugal potential associated with wobbles induced by the tesseral potential. The torque is thus computed on the static shape of the Earth, while the much smaller torque resulting from the action of the potential on the variations induced by the potential in the shape of the Earth is missing.

Several studies have investigated the second-order effects on the Earth's nutation due to the action of the tesseral potential $V_{21}$ on the time dependent increments to the Earth's flattening produced by the zonal part of the tidal potential. Studies by Souchay \& Folgueira (1999) yielded an estimated contribution of about $170 \mu$ as to the coefficient of the 18.6-yr nutation in longitude from the zonal tidal deformations. Their approach was to compute the increments to a rigid Earth nutation that would result on adding to the ellipticity of the rigid Earth the time dependent contributions to ellipticity from the zonal tidal deformations of a nonrigid Earth. Both MHB and Lambert \& Capitaine (2004) made computations of the same effect based on deformable Earth models with fluid core (with mantle anelasticity and ocean tide contributions to deformation taken into account in the former) and obtained results of the same order; MHB considered in addition the second order contribution to nutation from the action of the tesseral potential on deformations produced by the sectorial potential. Considering the precision of the nutation observations as well as the accuracies of the rigid Earth theories, it is clearly important that second order contributions of the order indicated above be carefully investigated and taken into account.

The torque exerted by the tesseral potential acting on tidal deformations is not the only second order effect to be considered. The torque due to the action of the zonal and sectorial parts of the 
potential on the deformations produced by other parts of the tidal potential are equally important, as will be shown here. In fact, Mathews (2003) had reported that the action of the zonal and sectorial potentials on the deformations due to the tesseral potential nearly cancels out the effects already considered by MHB (see also Escapa et al. 2004). The present work aims to clarify the influence of each second order term on precession-nutation. The contributions of ocean tides to the second order torque are systematically examined in this context.

The equations governing the perturbations of the rotational motion are given in Sect. 2 while Sect. 3 provides the expressions for the torque and explores the deformability of the Earth. The dynamical equations are solved in Sect. 4 and the results are discussed in Sect. 5.

\section{The Earth's rotation variations}

The variations in rotation of the Earth are governed by the angular momentum conservation law. In a frame attached to the rotating Earth,

$$
\frac{\mathrm{d} \boldsymbol{H}}{\mathrm{d} t}+\omega \times \boldsymbol{H}=\boldsymbol{\Gamma}
$$

where $\boldsymbol{H}$ is the angular momentum of the Earth, $\boldsymbol{\omega}$ is the instantaneous angular velocity vector, and $\boldsymbol{\Gamma}$ the external torque.

$\boldsymbol{H}=\underline{\bar{I}} \omega$,

where $\bar{I}$ is the inertia tensor. We use a reference frame with its axes oriented along the Earth's mean axes of inertia. Then the inertia tensor is

$\bar{I}=\left(\begin{array}{ccc}A & 0 & 0 \\ 0 & B & 0 \\ 0 & 0 & C\end{array}\right)+\left(\begin{array}{lll}c_{11} & c_{12} & c_{13} \\ c_{21} & c_{22} & c_{23} \\ c_{31} & c_{32} & c_{33}\end{array}\right)$

where the $c_{i j}$ represent the contributions to the elements of the inertia tensor from the deformations of the Earth due to various causes (e.g., tidal forces, surface loading, centrifugal forces due to Earth rotation variations). Following standard notation, we write the components of the angular velocity vector as $\omega_{1}=$ $\Omega m_{1}, \omega_{2}=\Omega m_{2}, \omega_{3}=\Omega\left(1+m_{3}\right)$ where $\Omega$ is the mean rotation rate of the Earth.

We consider an Earth model consisting of an anelastic mantle and a fluid core. We ignore the solid inner core as its effect on the second order contributions to nutations that we are seeking to calculate will be far too small to matter. The equatorial components $\left(m_{1}, m_{2}\right)$, or equivalently, their complex combination $\tilde{m}=m_{1}+i m_{2}$, represent the wobble of the mantle. We need to use also the similar quantities $\left(m_{1, \mathrm{f}}, m_{2, \mathrm{f}}\right)$ or $\tilde{m}_{\mathrm{f}}=m_{1, \mathrm{f}}+i m_{2, \mathrm{f}}$ to represent the differential wobble of the fluid core relative to the mantle (the subscript $\mathrm{f}$ is for "fluid"); $m_{3}$ and $m_{3, \mathrm{f}}$ stand for the fractional increment to the Earth's axial rotation rate and to that of the fluid core relative to the mantle. The equations for $\tilde{m}$, $\tilde{m}_{\mathrm{f}}, m_{3}$ and $m_{3, \mathrm{f}}$ (Sasao et al. 1980; Mathews et al. 1991) are:

$$
\begin{aligned}
\dot{\tilde{m}}-\mathrm{i} e \Omega \tilde{m}+\frac{\dot{\tilde{c}}+\mathrm{i} \Omega \tilde{c}}{A}+\frac{A_{\mathrm{f}}}{A}\left(\dot{\tilde{m}}_{\mathrm{f}}+i \Omega \tilde{m}_{\mathrm{f}}\right) & =\frac{\tilde{\Gamma}}{A \Omega} \\
\dot{\tilde{m}}+\dot{\tilde{m}}_{\mathrm{f}}-\mathrm{i} e_{\mathrm{f}} \Omega \tilde{m}_{\mathrm{f}}+\mathrm{i} \Omega \tilde{m}_{\mathrm{f}}+\frac{\dot{\tilde{c}}_{\mathrm{f}}}{A_{\mathrm{f}}} & =0 \\
\dot{m}_{3}+\frac{C_{\mathrm{f}}}{C} \dot{m}_{3, \mathrm{f}}+\frac{\dot{c}_{33}}{C} & =\frac{\Gamma_{3}}{C \Omega} \\
\dot{m}_{3, \mathrm{f}}+\frac{\dot{c}_{33, \mathrm{f}}}{C_{\mathrm{f}}} & =0
\end{aligned}
$$

where $e=(C-A) / A$ is the Euler frequency, $e_{\mathrm{f}}=\left(C_{\mathrm{f}}-A_{\mathrm{f}}\right) / A_{\mathrm{f}}$ and $\tilde{c}=c_{13}+i c_{23}, \tilde{c}_{\mathrm{f}}=c_{13, \mathrm{f}}+i c_{23, \mathrm{f}}, \tilde{\Gamma}=\Gamma_{1}+i \Gamma_{2}$.

The nutation angles $\Delta \psi$ and $\Delta \epsilon$ describing the motion of the Earth's figure axis in space are related to the solution obtainable for $\tilde{m}$ from Eqs. (4) and (5) through:

$\Delta \dot{\epsilon}-\mathrm{i} \Delta \dot{\psi} \sin \epsilon=-\Omega \tilde{m} \mathrm{e}^{\mathrm{i} \Phi}$

where $\Phi$ is the sidereal rotation angle.

\section{Torque on the tidal redistribution}

\subsection{The tidal potentials}

We use for the lunisolar potential of spherical harmonic order $l$ and degree $m$ a generalized form of the expression employed by Sasao et al. (1980) for the degree 2 tesseral $(l=2, m=1)$ potential. At any point within the Earth at geocentric distance $r$, terrestrial longitude $\lambda$ and colatitude $\theta$,

$V_{l m}(r, \theta, \lambda)=-\frac{1}{3} \Omega^{2} r^{2} \operatorname{Re}\left(\phi_{l m} Y_{l m}\right)$

where we define $Y_{l m}$, following Sasao et al. (1980), by $Y_{l m}=$ $P_{l m}(\cos \theta) \exp (-\mathrm{i} m \lambda)$, and

$\phi_{20}=K \frac{3 \sin ^{2} \beta-1}{2}$,

$\tilde{\phi}_{21}=\phi_{21}^{\mathrm{re}}+\mathrm{i} \phi_{21}^{\mathrm{im}}=K \sin \beta \cos \beta \mathrm{e}^{\mathrm{i} \Lambda}$,

$\tilde{\phi}_{22}=\phi_{22}^{\mathrm{re}}+\mathrm{i} \phi_{22}^{\mathrm{im}}=\frac{1}{4} K \cos ^{2} \beta \mathrm{e}^{2 \mathrm{i} \Lambda}$,

where $\beta$ and $\Lambda$ stand for the latitude and longitude of position of the perturbing celestial body referred to a terrestrial frame with its $x$ and $y$ axes along the equatorial principal axes of inertia, and $K=3 G M / d^{3} \Omega^{2}$ is dimensionless ( $M$ and $d$ are the mass and the geocentric distance of the perturbing body, respectively).

The apparent motion of the perturbing body from south to north of the equator and back (annual, in the case of the Sun and close to once a month in the case of the Moon) determines the frequency spectrum of $\phi_{20}$, which consists of low frequencies $(\ll \Omega)$. The apparent westward motions of the Sun and the Moon in the terrestrial frame causes $\mathrm{e}^{\mathrm{i} \Lambda}$ to have a frequency spectrum centered at $-\Omega$, the central frequency being due to the diurnal rotation of the Earth and the other frequencies being the result of the approximately periodic motions of the bodies in space. Consequently the spectrum of $\tilde{\phi}_{21}$ is in the retrograde diurnal band with frequencies $\sigma$ centered at $-\Omega$, and that of $\tilde{\phi}_{22}$ in the retrograde semidiurnal band. In the following, we will have to consider also $\tilde{\phi}_{21}^{*}$, which involves $\mathrm{e}^{-\mathrm{i} \Lambda}$; its frequently spectrum will evidently be in the prograde diurnal band, centered at $+\Omega$. It must be noted that the amplitudes $\tilde{\phi}_{21}(\sigma)$ of the spectral components of $\tilde{\phi}_{21}(t)$ are real, and so the amplitude of the term with frequency $-\sigma$ in $\tilde{\phi}^{*}(t)$ is the same as that of the term of frequency $\sigma$ in $\tilde{\phi}(t)$.

The zonal, tesseral and sectorial parts of the degree 2 potential correspond to $(l=2, m=0),(l=2, m=1)$ and $(l=2$, $m=2)$ respectively. The associated Legendre functions of degree 2 being:

$$
\begin{aligned}
& P_{20}(\cos \theta)=\frac{3 \cos ^{2} \theta-1}{2}, \\
& P_{21}(\cos \theta)=3 \cos \theta \sin \theta, \\
& P_{22}(\cos \theta)=3 \sin ^{2} \theta,
\end{aligned}
$$


one gets the following expressions for the potential in terms of the Cartesian coordinates $(x, y, z)$ :

$V_{20}=-\Omega^{2} \phi_{20} \frac{2 z^{2}-\left(x^{2}+y^{2}\right)}{6}$,
$V_{21}=-\Omega^{2}\left(\phi_{21}^{\mathrm{re}} x z+\phi_{21}^{\mathrm{im}} y z\right)$,
$V_{22}=-\Omega^{2}\left(\phi_{22}^{\mathrm{re}}\left(x^{2}-y^{2}\right)+2 \phi_{22}^{\mathrm{im}} x y\right)$.

\subsection{Incremental torques}

The incremental torque $\Delta \boldsymbol{\Gamma}_{l m}$ on the Earth due to the action of the potential $V_{l m}$ on the density perturbation $\delta \rho$ arising from the deformation produced by the potential is

$\Delta \boldsymbol{\Gamma}_{l m}=-\int \boldsymbol{r} \times \nabla V_{l m} \delta \rho \mathrm{d} v$

where the integral runs over the volume the Earth. For potentials of degree 2, one can readily evaluate this integral: on substituting from Eqs. (16)-(18) and noting that the increments $c_{i j}$ to the elements of the Earth's inertia tensor are defined by

$c_{i j}=\int\left(r^{2} \delta_{i j}-x_{i} x_{j}\right) \delta \rho \mathrm{d} v$

where the Kronecker function $\delta_{i j}$ is unity if $i=j$ and zero otherwise, one finds readily that the equatorial parts $\Delta \tilde{\Gamma}_{2 m}=$ $\Delta \Gamma_{2 m, 1}+\mathrm{i} \Delta \Gamma_{2 m, 2}$ of the torques $\Delta \boldsymbol{\Gamma}_{2 m}$ are given by

$\Delta \tilde{\Gamma}_{20}=-\mathrm{i} \Omega^{2} \phi_{20} \tilde{c}$

$\Delta \tilde{\Gamma}_{21}=-\Omega^{2} \phi_{21}^{\mathrm{im}}\left(c_{33}-c_{22}\right)+\mathrm{i} \Omega^{2} \phi_{21}^{\mathrm{re}}\left(c_{33}-c_{11}\right)$ $+\Omega^{2} \tilde{\phi}_{21}^{*} c_{12}$,

$\Delta \tilde{\Gamma}_{22}=2 \mathrm{i} \Omega^{2} \tilde{\phi}_{22} \tilde{c}^{*}$,

where $\tilde{c}=c_{13}+\mathrm{i} c_{23}$. The $j$ th component of $\Delta \boldsymbol{\Gamma}_{2 m}$ has been denoted above by $\Delta \Gamma_{l m, j}$.

Note that the relative variations of the moments of inertia $c_{i j} / C$ are typically $10^{-8}$.

\subsection{Deformability of the Earth, and the increments of inertia}

The increment to the Earth's own gravitational potential $V_{\mathrm{E}}$ that results from the redistribution of mass caused by the deformation of the Earth due to the direct action of an external potential $V_{l m}(r, \theta, \lambda)$ is proportional to this potential. The proportionality factor is the Love number $k_{l m}$ which is a measure of the effect of the deformability of the Earth on the Earth's external gravitational potential. One has, at points on the surface $r=a, a$ being the mean equatorial radius of the Earth,

$\delta V_{\mathrm{E}, l m}=k_{l m} V_{l m}$.

For $l=2$, the redistribution potential $\delta V_{\mathrm{E}, 2 m}$ is linked to the moments of inertia of the redistribution via McCullagh's theorem (McCullagh 1855; see also Munk \& McDonald 1960). Indeed, one has:

$\delta V_{\mathrm{E}(l=2)}=-\frac{G}{2 r^{5}}\left(c_{k k} r^{2} \delta_{i j}-3 c_{i j} x_{i} x_{j}\right)$

wherein the $x_{i}$ pertain to any point outside the body.

Now, introduction of the $V_{2 m}$ from Eqs. (16)-(18) into Eq. (25) leads to expressions in terms of Cartesian coordinates for the $\delta V_{\mathrm{E}, 2 m}$. Taking the sum over $m$ and comparing the resulting expression to (24), one gets the increments of inertia due to deformations caused by the direct action of the degree 2 tidal potential:

$$
\begin{aligned}
c_{11}^{(\mathrm{d})} & =\frac{1}{3} \kappa_{20} A \phi_{20}-2 \kappa_{22} A \phi_{22}^{\mathrm{re}}, \\
c_{22}^{(\mathrm{d})} & =\frac{1}{3} \kappa_{20} A \phi_{20}+2 \kappa_{22} A \phi_{22}^{\mathrm{re}}, \\
c_{33}^{(\mathrm{d})} & =-\frac{2}{3} \kappa_{20} A \phi_{20}, \\
c_{12}^{(\mathrm{d})} & =-2 \kappa_{22} A \phi_{22}^{\mathrm{im}}, \\
c_{13}^{(\mathrm{d})} & =-\kappa_{21} A \phi_{21}^{\mathrm{re}}, \\
c_{23}^{(\mathrm{d})} & =-\kappa_{21} A \phi_{21}^{\mathrm{im}},
\end{aligned}
$$

where $\kappa_{l m}=k_{l m} a^{2} \Omega^{2} / 3 G A$ and the superscript $(d)$ stands for "direct". The compliances $\kappa_{l m}$ are real for an elastic Earth; they become complex when mantle anelasticity is taken into account. The ellipticity and rotation of the Earth as well as anelasticity give rise to small differences among the values of the $\kappa_{2 m}$ for $m=0,1,2$.

Indirect contributions to the inertia tensor arise from the deformations caused by the centrifugal forces associated with Earth rotation variations and by the redistribution of ocean mass (ocean tides) produced by the potential. They will be considered in the next section. The total $c_{i j}$ is the sum of the direct and indirect effects; and it is the use of this $c_{i j}$ rather than $c_{i j}^{(\mathrm{d})}$ in Eq. (25) that leads to the observable $\delta V_{\mathrm{E}(l=2)}$.

It may be noted from Eqs. (26)-(31) that $c_{11}$ and $c_{22}$ get contributions from both zonal and sectorial excitations:

$$
\begin{aligned}
& c_{11}=c_{11}^{z}+c_{11}^{s} \\
& c_{22}=c_{22}^{z}-c_{22}^{s} \\
& c_{22}^{z}=c_{11}^{z}=-\frac{1}{2} c_{33} \\
& c_{22}^{s}=-c_{11}^{s}
\end{aligned}
$$

where the superscripts $z$ and $s$ identify the zonal and sectorial parts. Each of the other elements is generated by the action of a potential of a single order: $c_{13}$ and $c_{23}$ by the tesseral potential only and $c_{33}$ by the zonal part alone. These properties are evident from the expressions given for the direct part of the $c_{i j}$; they hold, in fact, for the full $c_{i j}$. Use of these relations enables us now to rewrite $\Delta \tilde{\Gamma}_{21}$ of Eq. (22) as:

$\Delta \tilde{\Gamma}_{21}=\frac{3}{2} \mathrm{i} \Omega^{2} c_{33} \tilde{\phi}_{21}-\mathrm{i} \Omega^{2}\left(c_{11}^{s}+\mathrm{i} c_{12}\right) \tilde{\phi}_{21}^{*}$.

Since $c_{33}$ is generated solely by the zonal potential, the first term in this expression represents the torque due to the action of the tesseral potential on the deformation produced by the zonal potential; Eq. (21) shows that that $\Delta \tilde{\Gamma}_{20}$ is the result of the reciprocal effect: the action of the zonal potential on the deformation caused by the tesseral potential. Similarly the second term of (36) represents the interaction of the tesseral potential with the deformation due to the sectorial potential, while the expression (23) for $\Delta \tilde{\Gamma}_{22}$ represents the reciprocal action.

We noted in Sect. 3.1 the nature of the spectra of zonal, tesseral, and sectorial potentials. Furthermore, the deformation caused by each type of potential has the same spectrum as the potential. The product of $c_{33}$, which is due to the zonal potential having a low frequency spectrum, with $\tilde{\phi}_{21}$ having a retrograde diurnal specturm evidently produces a retrograde diurnal spectrum for the first term of the above expression for $\Delta \tilde{\Gamma}_{21}$; 
the same is true for the second term which is a product of retrograde semidiurnal and prograde diurnal factors. One sees similarly from Eqs. (21) and (23) that the spectra of $\Delta \tilde{\Gamma}_{20}$ and $\Delta \tilde{\Gamma}_{22}$ too are in the retrograde diurnal band. These statements need to be qualified when the ocean tide contribution is also taken into account, because the ocean tides generated by the retrograde diurnal/semidurnal potential waves contain prograde diurnal/semidiurnal waves too besides the retrograde ones, as will be seen in Sect. 3.5. For example, the ocean tide contribution to $c_{11}^{s}+\mathrm{i} c_{12}$ from the prograde part of the ocean tide raised by the sectorial potential will be prograde semidiurnal, and when it is multiplied by $\tilde{\phi}_{21}^{*}$ the resulting torque is prograde terdiurnal. However the nutation produced by it is of negligible magnitude, and so we need consider only the retrograde part of the ocean tides. Similar statements hold for the effects of the ocean tides produced by the potentials of other orders.

The sum of the three torques (21), (23), and (36) would reduce to zero, as observed by Mathews (2003) and Escapa et al. (2004), if the $c_{i j}$ consisted of only the direct terms (26)-(31) and if $\kappa_{20}, \kappa_{21}, \kappa_{22}$ were all real and had equal values. Actually, the values are complex and unequal as a consequence of anelasticity, ellipticity, and the Coriolis force due to Earth rotation; and the centrifugal and ocean tide contributions to the $c_{i j}$ give rise to further frequency dependent differences. Therefore there exists a residual second order torque which leads to non-negligible contributions to the nutations at certain periods and to the precession, as will be seen below.

\subsection{Increments of inertia, including ocean tidal effects}

We need to consider now the contributions to the incremental inertia tensor $c_{i j}$ from the centrifugal forces due to Earth rotation variations and from the deformation caused by ocean tidal loading; these, taken together with the contribution from the direct action of the potentials, shown in Eqs. (26)-(31), give us the total $c_{i j}$. We take up these effects separately for the excitations due to the three types of potentials.

The contribution from the effects of the tesseral potential may be expressed as

$\tilde{c}=-A\left[\kappa_{21}\left(\tilde{\phi}_{21}-\tilde{m}\right)-\xi_{21} \tilde{m}_{\mathrm{f}}\right]+\tilde{c}^{(\mathrm{OT})}$,

where the last term is the ocean tidal contribution (as is evident from the notation) and the other terms are as in Sasao et al. (1980). The term involving $\tilde{\phi}_{21}$ represents the direct effect $\tilde{c}^{(\mathrm{d})}$, while the terms proportional to $\tilde{m}$ and $\tilde{m}_{\mathrm{f}}$ arise from the centrifugal forces due to the wobble of the mantle and the differential wobble of the fluid core, respectively. The contribution of these centrifugal terms is quite significant for some of the nutations. While $\tilde{m}$ is small, of order $e \tilde{\phi}_{21}, \tilde{m}_{\mathrm{f}}$ is about 200 times $\tilde{m}$ for the wobble corresponding to the retrograde 18.6-yr nutation, and $\xi / \kappa \approx 0.22$, and so its contribution comes to about $14 \%$ of the direct term. (The existence of the Nearly Diurnal Free Wobble eigenmode with the retrograde period of approximately -430 days causes resonant enhancement of $\tilde{m}$ and far greater enhancement of $\tilde{m}_{\mathrm{f}}$ at nearby frequencies.) MHB expressed the spectral components $\tilde{c}^{(\mathrm{OT})}(\sigma)$ of the ocean tide contribution, following Sasao \& Wahr (1981), as $\tilde{c}^{(\mathrm{OT})}(\sigma)=-A \Delta \kappa^{(\mathrm{OT})} \tilde{\phi}_{21}$, and obtained the ocean tidal increments to $\kappa_{21}$, namely $\Delta \kappa^{\mathrm{OT}}$, with the help of available data on the ocean tidal angular momentum. We have employed this method to evaluate $\tilde{c}^{(\mathrm{OT})}(\sigma)$ for frequencies of interest to our calculation of second order contributions to the nutations. We have also used programs of MHB to evaluate the the square-bracketed factor in Eq. (37) for these frequencies, thereby completing the evaluation of $\tilde{c}$.
For a sectorial excitation, one has $c_{11}^{s}=-c_{22}^{s}$, where the superscript $s$ stands for the sectorial deformations only. Rotation variations produced by the sectorial potential are proportional to the triaxiality parameter which is of order $10^{-5}$, and therefore the associated centrifugal effects are quite ignorable. Consequently one may drop the superscript (d) from terms involving the sectorial potential in Eqs. (26), (27), and (29). On combining them, we have:

$c_{11}^{s}+\mathrm{i} c_{12}^{s}=-2 \kappa_{22} A \tilde{\phi}_{22}+\left(c_{11}^{s}+\mathrm{i} c_{12}^{s}\right)^{(\mathrm{OT})}$.

We have used the data (from the CSR4 ocean tide model) on the amplitudes $C_{s, 22}^{ \pm}$and $S_{s, 22}^{ \pm}$of the spectral components of ocean tides of spherical harmonic type $(l=2, m=2)$ raised by the sectorial potentials of various frequencies to arrive at the spectral amplitudes of the ocean tide part of the above expression. The relations of the $c_{i j}^{(\mathrm{OT})}$ to the tide height amplitudes are presented in the next section. The value used for $\kappa_{22}$ was obtained by conversion (multiplication by $a^{2} \Omega^{2} / 3 G A$ ) from the nominal value $k_{22}=0.30102-0.00130 \mathrm{i}$ shown in the IERS Conventions 2003, Chapter 6.

As for the deformations excited by zonal tides, a direct measure of $c_{33}$ is provided by the deviation $m_{3} \Omega$ of the axial rotation rate from the mean rate $\Omega$, which is reflected in the length-of-day (LOD) variations. Accurate modeling of the LOD variations (e.g., by Defraigne \& Smits 1999) recognizes the nonparticipation of the core in the axial rotation variations and also takes account of the contributions of anelasticity and ocean tides. One has then $m_{3}=c_{\mathrm{m}, 33} / C_{\mathrm{m}}$ where the subscript $\mathrm{m}$ refers to the mantle; the increment $c_{33}$ for the whole Earth may therefore be expressed, following MHB, as

$\begin{aligned} c_{33} & =-C_{\mathrm{eff}} m_{3}^{z}, \\ C_{\mathrm{eff}} & =\frac{C_{\mathrm{m}}}{1-\gamma_{20} C_{\mathrm{f}} / \kappa_{20} C},\end{aligned}$

where $C_{\mathrm{m}}=C-C_{\mathrm{f}}$ is the axial moment of inertia of the mantle, and $\kappa_{20}$ and $\gamma_{20}$ represent compliances of the whole Earth and the core, respectively, for the zonal tidal potential. (It turns out that $C_{\text {eff }}=1.2711 C_{\mathrm{m}}$.) The spectral amplitudes of $m_{3} \Omega$ may be found listed in Chapter 8 of the IERS Conventions 2003; we have used them along with the above equations to compute the spectral components of $c_{33}$. Incidentally, the centrifugal contribution to $c_{33}$ is $(4 / 3) A \kappa_{20} m_{3}$, which is only about $10^{-4}$ times the direct part given by Eq. (31) and its effect on nutation is entirely negligible.

\subsection{Ocean tidal increments to geopotential coefficients and the $c_{i j}$}

The ocean tide raised by the tidal potential involves a redistribution of the ocean water mass. The loading of the crust by this incremental mass distribution (which is nonzero only over the oceans), as well as the attraction of the matter in the Earth's interior by this mass, causes deformations which affect the inertia tensor of the Earth and the core regions, and the resultant perturbations of the Earth's gravitational field.

The ocean tidal contribution to the $(l m)$ part of the Earth's gravitational potential $V_{\mathrm{E}}$ is:

$$
\begin{aligned}
\Delta V_{\mathrm{E}, l m}^{(\mathrm{OT})}=-\frac{G M}{a} \operatorname{Re}[ & \left(\Delta C_{l m}^{(\mathrm{OT})}+\mathrm{i} \Delta S_{l m}^{(\mathrm{OT})}\right) \\
& \left.\times P_{l m}(\cos \theta) \mathrm{e}^{-i m \lambda}\right]
\end{aligned}
$$


Expressions relating the ocean tidal increments $\Delta C_{l m}^{(\mathrm{OT})}$ and $\Delta S_{l m}^{(\mathrm{OT})}$ to the geopotential coefficients are given in Chapter 6 of IERS Conventions (2003) in terms of the spectral amplitudes $C_{s l m}^{ \pm}$and $S_{s l m}^{ \pm}$of the cosine and sine parts of the tide height for the tidal constituent $s$ :

$$
\begin{aligned}
\Delta C_{l m}^{(\mathrm{OT})}+i \Delta S_{l m}^{(\mathrm{OT})}= & L_{l m} \sum_{s(l m)}\left[\left(C_{s l m}^{+}+\mathrm{i} S_{s l m}^{+}\right) \mathrm{e}^{-\mathrm{i} \Theta_{s}}\right. \\
& \left.+\left(C_{s l m}^{-}-i S_{s l m}^{-}\right) \mathrm{e}^{i \Theta_{s}}\right]
\end{aligned}
$$

where $\Theta_{s}$ is the argument of the tidal constituent $s$, and

$L_{l m}=\left(\frac{4 \pi G \rho_{\mathrm{w}}}{g}\right)\left(\frac{1+k_{l m}^{\prime}}{2 l+1}\right) K_{l m}$

with

$K_{l m}=\left(\frac{(2 l+1)(l-m) !\left(2-\delta_{m 0}\right)}{(l+m) !}\right)^{1 / 2}$

where $\rho_{\mathrm{w}}$ is the density of sea water. The part containing the factor $\mathrm{e}^{\mathrm{i} \Theta_{s}}$ represents a prograde wave with a prograde diurnal spectrum. As was explained in the last section, this part is ignorable for our purposes. The amplitudes that we need then are $C_{s l m}^{+}$ and $S_{s l m}^{+}$; their values may be taken from tables of ocean tide models (e.g., CSR4). The OT contributions to the increments of inertia can then be deduced from the equations above using the relationships between $c_{l m}^{(\mathrm{OT})}$ and the increments $\Delta C_{l m}$ that they produce to the geopotential coefficients:

$$
\begin{aligned}
c_{11}^{s(\mathrm{OT})}+i c_{12}^{(\mathrm{OT})} & =-2 M a^{2}\left(\Delta C_{22}^{(\mathrm{OT})}+i \Delta S_{22}^{(\mathrm{OT})}\right), \\
\tilde{c}^{(\mathrm{OT})} & =-M a^{2}\left(\Delta C_{21}^{(\mathrm{OT})}+i \Delta S_{21}^{(\mathrm{OT})}\right), \\
c_{33}^{(\mathrm{OT})} & =-\frac{2}{3} M a^{2} \Delta C_{20}^{(\mathrm{OT})} .
\end{aligned}
$$

Note that the tabulated OT data are with reference to the terrestrial frame with its $x$ axis in the Greenwich meridian, while our dynamical equations are expressed in the frame defined by the principal axes of inertia. The latter frame is rotated to the West by 14.95 relative to the former (see Bretagnon et al. 1997). We have taken account of this difference between the two frames in making our calculations relating to the sectorial tides. As was mentioned in the last section, the OT increments in the zonal and tesseral cases were calculated by other means.

As may be seen from MHB paper, the amplitude of the combined contribution $\Delta \kappa$ for the 18.6-yr term due to anelasticity and oceanic tides is about $8 \%$ of the amplitude of the elastic value of $\kappa$ (the real part only accounts for about $4 \%$ ), so that these effects are comparable in magnitude to the resonance effects mentioned earlier.

\section{Solution of the dynamical equations}

To make the dynamical Eqs. (4) and (5) for $\tilde{m}$ and $\tilde{m}_{\mathrm{f}}$ more explicit, we substitute for $\tilde{c}$ and $\tilde{c}_{\mathrm{f}}$ the expressions $\tilde{c}=-A\left[\kappa\left(\phi_{21}-\right.\right.$ $\left.\tilde{m})-\xi \tilde{m}_{\mathrm{f}}\right]$ and $\tilde{c}_{\mathrm{f}}=-A\left[\gamma\left(\phi_{21}-\tilde{m}\right)-\beta \tilde{m}_{\mathrm{f}}\right]$, where the former is of the same form as (37) but now with $\kappa=\kappa_{21}+\Delta \kappa^{(\mathrm{OT})}\left(\kappa_{21}\right.$ itself being taken for the anelastic Earth), and the expression for the incremental inertia of the fluid core is analogous. When the equations which emerge after the substitutions are written in the frequency domain, we obtain:

$$
\begin{aligned}
\alpha_{1 \mathrm{~m}} \tilde{m}(\sigma)+\alpha_{2 \mathrm{~m}} \tilde{m}_{\mathrm{f}}(\sigma) & =\tilde{\Gamma}(\sigma)+\sigma^{\prime} \kappa \tilde{\phi}_{21}(\sigma), \\
\alpha_{1 \mathrm{f}} \tilde{m}(\sigma)+\alpha_{2 \mathrm{f}} \tilde{m}_{\mathrm{f}}(\sigma) & =\sigma \gamma \tilde{\phi}_{21}(\sigma),
\end{aligned}
$$

Table 1. Frequency bands (in cycle per day) in the terrestrial frame of the variable quantities involved in the expressions (21), (36), and (23) for the torques $\Delta \tilde{\Gamma}_{20}, \Delta \tilde{\Gamma}_{21}$ and $\Delta \tilde{\Gamma}_{22}$. Boxes for cross terms which are not present in these expressions are left blank. 0 stands for the long period band.

\begin{tabular}{lrrrrr}
\hline \hline & & $\phi_{20}$ & $\tilde{\phi}_{21}$ & $\tilde{\phi}_{21}^{*}$ & $\tilde{\phi}_{22}$ \\
& & 0 & -1 & +1 & -2 \\
\hline$\phi_{20}, \tilde{m}_{3}^{z}$ & 0 & & -1 & & \\
$\tilde{\phi}_{21}, \tilde{m}, \tilde{m}_{\mathrm{f}}$ & -1 & -1 & & & \\
$\tilde{\phi}_{21}^{*}, \tilde{m}^{*}, \tilde{m}_{\mathrm{f}}^{*}$ & +1 & & & & -1 \\
$\tilde{\phi}_{22}$ & -2 & & & -1 & \\
\hline
\end{tabular}

where

$$
\begin{aligned}
\alpha_{1 \mathrm{~m}} & =\sigma+\kappa \sigma^{\prime}-e, & \alpha_{2 \mathrm{~m}} & =\sigma^{\prime}\left(\xi+A_{\mathrm{f}} / A\right), \\
\alpha_{1 \mathrm{f}} & =\sigma(1+\gamma), & \alpha_{2 \mathrm{f}} & =\sigma^{\prime}+\beta \sigma+e_{\mathrm{f}} .
\end{aligned}
$$

Here $\sigma^{\prime}=\sigma+\Omega$ is the frequency in the space-fixed reference frame.

Let us suppose that $\tilde{\Gamma}(\sigma)$ in the above equations is the usual first order torque. Incrementing it by $\Delta \tilde{\Gamma}(\sigma)$ (which is the spectral component of a second order torque $\Delta \tilde{\Gamma}(t))$ causes the solutions for the wobbles of the mantle and the core to be incremented by amounts $\delta \tilde{m}(\sigma)$ and $\delta \tilde{m}_{\mathrm{f}}(\sigma)$ respectively. It is evident that they satisfy the equations

$$
\begin{aligned}
\alpha_{1 \mathrm{~m}} \delta \tilde{m}(\sigma)+\alpha_{2 \mathrm{~m}} \delta \tilde{m}_{\mathrm{f}}(\sigma) & =\delta \tilde{\Gamma}(\sigma), \\
\alpha_{1 \mathrm{f}} \delta \tilde{m}(\sigma)+\alpha_{2 \mathrm{f}} \delta \tilde{m} \mathrm{f}(\sigma) & =0 .
\end{aligned}
$$

What is of interest to us for computing the increments to the nutation amplitudes is $\delta \tilde{m}(\sigma)$, which is trivially obtained from the above equations:

$$
\delta \tilde{m}(\sigma)=-\frac{\mathrm{i} \alpha_{2 \mathrm{f}}}{\alpha_{2 \mathrm{f}} \alpha_{1 \mathrm{~m}}-\alpha_{1 \mathrm{f}} \alpha_{2 \mathrm{~m}}} \Delta \Gamma(\sigma) .
$$

We may now take for $\Delta \tilde{\Gamma}(t)$ either $\Delta \tilde{\Gamma}_{20}(t)$ or $\Delta \tilde{\Gamma}_{22}(t)$ or either of the terms in the expression for $\Delta \tilde{\Gamma}_{21}(t)$ from Eqs. (21), (23), and (36). Each of these is a product of the form $p(t) q(t)$. So its spectral component having frequency $\sigma$ is $\sum_{\sigma^{\prime}} p\left(\sigma^{\prime}\right) q\left(\sigma-\sigma^{\prime}\right)$. It may be observed that one of the two factors relates to increments of inertia and the other factor to the amplitude $\tilde{\phi}$ of one or the other of the three types of tidal potentials. The spectral components of the relevant increments of inertia were evaluated as explained in Sect. 3.4. The spectral amplitudes of the tidal potentials were computed using the lunar theory ELP 2000 (ChaprontTouzé \& Chapront 1983) and the solar system semi-analytical solution VSOP87 (Bretagnon \& Francou 1988). We have used the recommended standard values (IERS Conventions 2003) for other parameters. The mean obliquity of the ecliptic at J2000.0 is $\epsilon_{0}=86431^{\prime \prime} 406$.

The values used for the compliances in Eqs. (50) and (51) were: $\kappa=0.0010340, \gamma=0.0019662$ which are the estimates from MHB, and $\xi=0.0002222$ and $\beta=0.0006160$ from Mathews et al. (1991). While the increments to these from anelasticity and ocean tides are important in the calculation of $\Delta \tilde{\Gamma}(\sigma)$, they are of no significance in the evaluation of $\delta \tilde{m}(\sigma)$ from Eq. (54), since the factor $\Delta \tilde{\Gamma}(\sigma)$ is already of the second order. Once $\delta \tilde{m}(\sigma)$ is evaluated for various frequencies, the second order corrections to the (prograde/retrograde) nutations having the corresponding frequencies $\sigma^{\prime}$ are obtained as

$\delta \tilde{\eta}\left(\sigma^{\prime}\right)=-\frac{1}{\sigma^{\prime}} \delta \tilde{m}(\sigma)$ 
Table 2. Contribution in the nutation ( $\mu$ as) due to the interaction between different type of tides and potentials. Boxes with values below $1 \mu$ as are left blank. EL: Elastic Earth, AE: Anelastic Earth, OT: Ocean Tides.

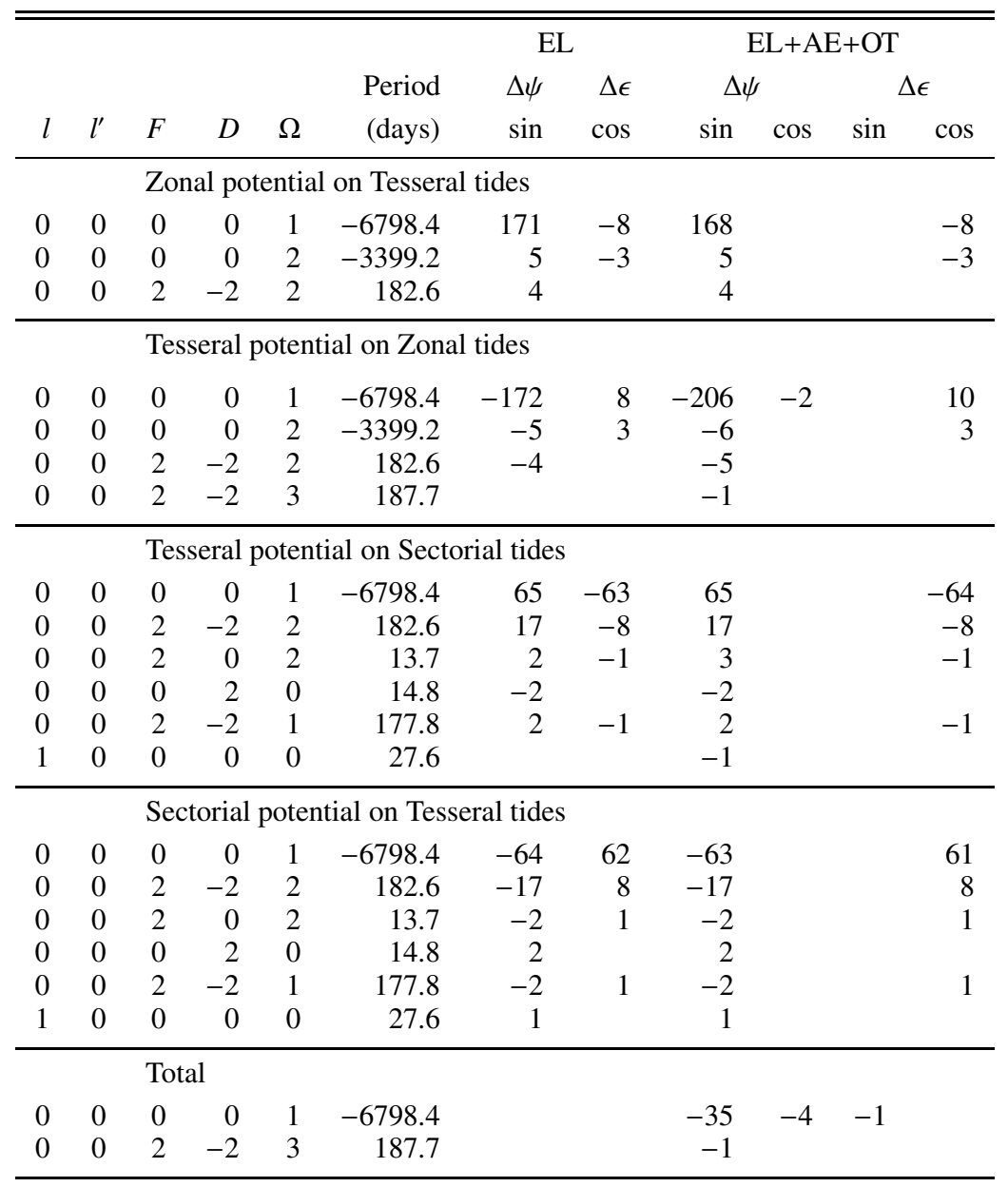

Table 3. Contribution in the precession ( $\mu$ as/cy) due to the interaction between different type of tides and potentials. Boxes with values below $1 \mu$ as are left blank. EL: Elastic Earth, AE: Anelastic Earth, OT: Ocean Tides.

\begin{tabular}{|c|c|c|c|c|c|}
\hline \multirow[b]{2}{*}{ Potential } & \multirow[b]{2}{*}{ Tides } & \multicolumn{2}{|l|}{ EL } & \multicolumn{2}{|c|}{$\mathrm{EL}+\mathrm{AE}+\mathrm{OT}$} \\
\hline & & $\psi$ & $\epsilon$ & $\psi$ & $\epsilon$ \\
\hline Zonal & Tesseral & -4104 & & -4045 & 9 \\
\hline Tesseral & Zonal & 4110 & & 4910 & 12 \\
\hline Tesseral & Sectorial & -27312 & & -27588 & 47 \\
\hline Sectorial & Tesseral & 27009 & & 26618 & 58 \\
\hline Total & & -297 & & -105 & 127 \\
\hline
\end{tabular}

The contributions to the nutation coefficients in longitude and obliquity are obtained in the usual fashion from the amplitudes of the corresponding pair of prograde and retrograde amplitudes.

Results of the computation are expressed as series of periodical terms. The phase of each term is a linear combination of Delaunay's fundamental arguments $\left(l, l^{\prime}, F, D, \Omega\right)$. Second order contributions larger than $1 \mu$ as to the nutation coefficients are gathered in Tables 2 (nutation) and 3 (precession). Note that most of the out-of-phase terms are not displayed for the anelastic plus OT part, since, for each individual tidal spectral component, they are only at the level of a few tenths of microarcsecond. However, their cumulated effect reaches $4 \mu$ as.

\section{Conclusion}

This study investigates the effects resulting from the coupling of each part of the degree 2 potential to deformations due to other parts of the potential. It is clear that the net effect is very small as a result of reciprocal cancellations: the effects of the tesseral potential on zonal deformations are nearly canceled out by the reciprocal effects of the zonal potential on tesseral deformations. In the same way, the effects of the tesseral potential on sectorial tides are almost canceled out by the effects of the sectorial potential on tesseral tides. The reasons for incomplete cancellation are that (i) the value of the compliance $\kappa$ differs for tides of different orders $(0,1,2)$ even for a nondissipative Earth, and (ii) for an anelastic Earth with oceans, the contributions from these to $\kappa$ are not only frequency dependent (with a different dependence in different frequency bands) but also complex, meaning that the response to tidal forcing is out of phase with the forcing.

The net effect on the nutation reaches $-35 \mu$ as on the 18.6-yr nutation in longitude and comes mainly from the oceanic tides. The effects found on the precession are consistent with those in MHB ( $-21 \mathrm{mas} / \mathrm{cy}$ for the cumulated effects of the tesseral potential on zonal and sectorial tides). The total effect on the precession is of the same order of magnitude ( $0.1 \mathrm{mas} / \mathrm{cy})$ in longitude and in obliquity. 
Acknowledgements. The authors are grateful to Srinivas Bettadpur of the Center for Space Research, University of Texas, for his help with tables of ocean tides. They thank also the referee for his careful review of the manuscript.

\section{References}

Bretagnon, P., \& Francou, G. 1988, A\&A, 202, 309

Bretagnon, P., Rocher, P., \& Simon, J.-L. 1997, A\&A, 319, 305

Chapront-Touzé, M., \& Chapront, J. 1983, A\&A, 124, 50

Defraigne, P., \& Smits, I. 1999, Geophys. J. Int., 139, 563

Dehant, V., Feissel-Vernier, M., de Viron, O., et al. 2003, J. Geophys. Res., 108, B5

Escapa, A., Getino, J. M., \& Ferrandiz, J. M. 2004, Proc. Journées Syst. Ref., ed. N. Capitaine, Paris, 70

Hefty, J., \& Capitaine, N. 1990, Geophys. J. Int., 103, 219

IERS Conventions 2003, IERS Technical Note 32, ed. D. D. McCarthy, \& G. Petit, Bundesamt für Kartographie und Geodäsie, Frankfurt am Main
Lambert, S., \& Capitaine, N. 2004, A\&A, 428, 255

McCullagh, J. 1855, R. Irish Acad., 22, 139

Mathews, P. M. 2003, American Geophysical Union, Fall Meeting 2003, abstract \#G21A-05

Mathews, P. M., \& Bretagnon, P. 2003, A\&A, 400, 1113

Mathews, P. M., Buffett, B. A., Herring, T. A., \& Shapiro, I. I. 1991, J. Geophys. Res., 96, B5, 8243

Mathews, P. M., Herring, T. A., \& Buffett, B. A. 2002, J. Geophys. Res., 107, B4

Munk, W. H., \& McDonald, G. 1960, The Rotation of the Earth (Cambridge University Press)

Roosbeek, F., \& Dehant, V. 1998, Celest. Mech. Dyn. Astr., 70 (4), 215

Sasao, T., \& Wahr, J. M. 1981, Geophys. J. R. Astron. Soc., 64, 729

Sasao, T., Okubo, S., \& Saito, M. 1980, Proc. IAU Symp. 78, ed. E. P. Federov, M. L. Smith, \& P. L. Bender (Hingham, Mass.: D. Reidel), 165

Seidelmann, P. K. 1982, Celest. Mech., 27, 79

Souchay, J., \& Folgueira, M. 1999, EM\&P, 81, 201

Souchay, J., Loysel, B., Kinoshita, H., \& Folgueira, M. 1999, A\&AS, 135, 111

Yoder, C. F., Williams, J. G., \& Parke, M. E. 1981, J. Geophys. Res., 86, 881 